\title{
Probing the Tri-Linear Higgs Self-Coupling
}

\author{
Austin Grenert, Elgin Community College, CCI Program \\ Mia Liu and Aashaq Shah, Fermilab \\ On behalf of the CMS Collaboration
}

One of the leading feats in modern physics is to further study and measure the most recent edition to the Standard Model (SM), the Higgs Boson. Discovered in 2012, The Higgs boson is one of the fundamental particles in the SM. It is produced by one of the most important field in particle physics theories, the Higgs Field. The Higgs boson is the visible manifestation of the Higgs field [3]. Interaction with the Higgs Field is what causes other SM particles as well as the $\mathrm{W}$ and $\mathrm{Z}$ bosons to have mass.

\section{Project Goals}

The goal of this project is to study the feasibility of probing tri-linear Higgsself coupling in a process where a single Higgs is produced in vector boson scattering associated with two additional protons, i.e. , where the $W$ bosons decay leptonically and the Higgs decays to $b \bar{b}$. The leptons are required to have the same charge in order to suppress other standard model backgrounds. Any deviations of the Higgs-self coupling from the value predicted by the SM could be indications of Beyond the Standard Model (BSM) physics.

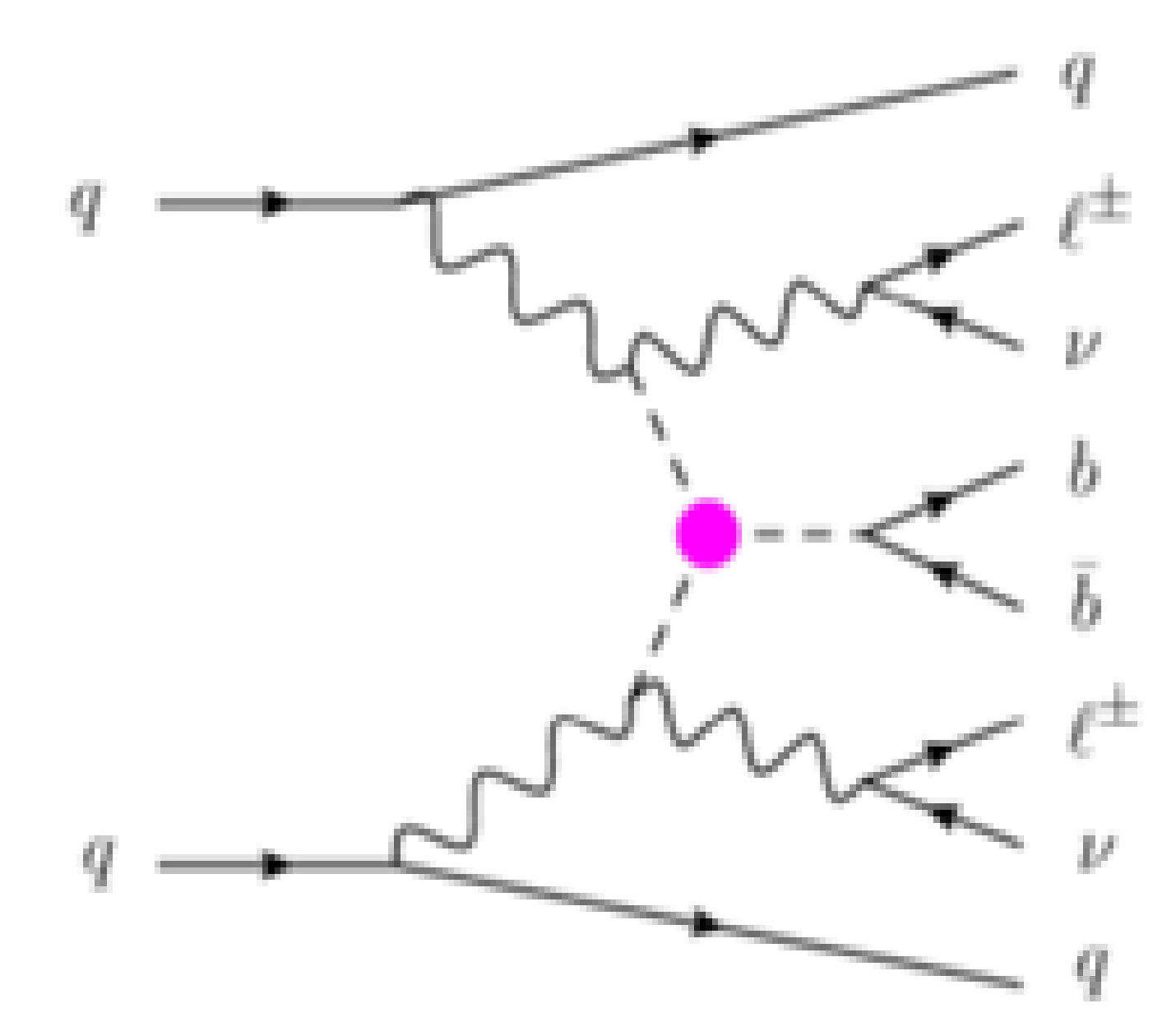

Figure 1: Diagram of the tri-linear Higgs self-coupling.

\section{Methodology}

In order to study this process we must first look at a selection of standard model equations.

Higgs Potential: $\quad-V(H)=\frac{1}{2} m_{H} H^{2}+\lambda_{3} v H^{3}+\frac{1}{4} \lambda_{4} H^{4}+O\left(H^{5}\right)$, - $V^{S M}(\Phi)=-\mu^{2}\left(\Phi^{\dagger} \Phi\right)+\lambda\left(\Phi^{\dagger} \Phi\right)^{2}$,

where $\mu^{2}=\frac{m_{H}^{2}}{2}$ and $\lambda=\frac{m_{H}^{2}}{2 v^{2}}, m_{H}=125 \mathrm{GeV}, v \simeq 246 \mathrm{GeV}$. [2]

- Through Electro-Weak Symmetry Breaking (EWSB) in equation (2), the mass of the Higgs is determined, and thus the standard model completely determines the Higgs self-coupling constant to be

$$
\lambda_{3}^{S M}=\lambda_{4}^{S M} \simeq 0.13 \text {. [2] }
$$

- In the case of the trilinear coupling and at the order we are considering, one-loop corrections for single Higgs processes, adding higher dimensional operators that only affect the Higgs self couplings can directly be introduced via an anomalous coupling

$$
\lambda_{3}=\kappa_{3} \lambda_{3}^{S M} \text {. [2] }
$$

- From this modified coupling, the formula for the $\lambda_{3}$ dependence of a generic NLO observable $\Sigma_{\lambda_{3}}^{B S M}$ can be written as

$$
\begin{gathered}
\sum_{\lambda_{3}}^{B S M}=Z_{H}^{B S M} \Sigma_{L O}\left(1+\kappa_{3} C_{1}+\delta Z_{H}\right), \\
Z_{H}^{B S M}=\frac{1}{1-\left(\kappa_{3}^{2}-1\right) \delta Z_{H}}, \quad \delta Z_{H}=-1.536 \times 10^{-3}
\end{gathered}
$$

- $C_{1}$ is the process and kinematic dependent component (Shown in Figure 3).

- $\Sigma_{L O}$ is the leading order prediction value for the observable (in our case cross-section).

- Significant deviations in $\lambda_{3}$ due to the value (currently unknown) of anomalous coupling $\kappa_{3}$ can be an indication of a new discovery of BSM physics, traditionally deviations must be at or exceed $\pm 5 \sigma$.

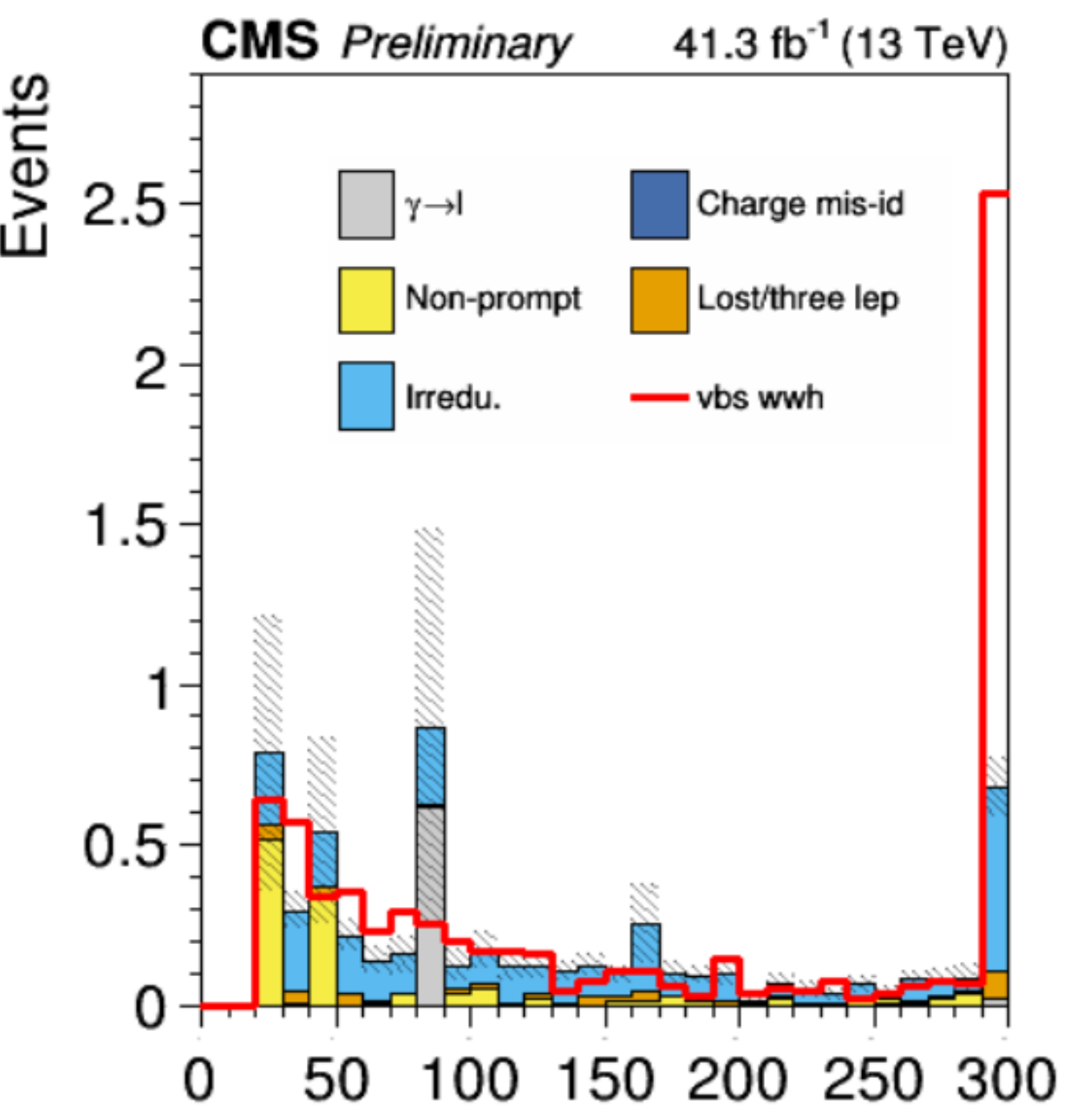

(A) bjet_pt1

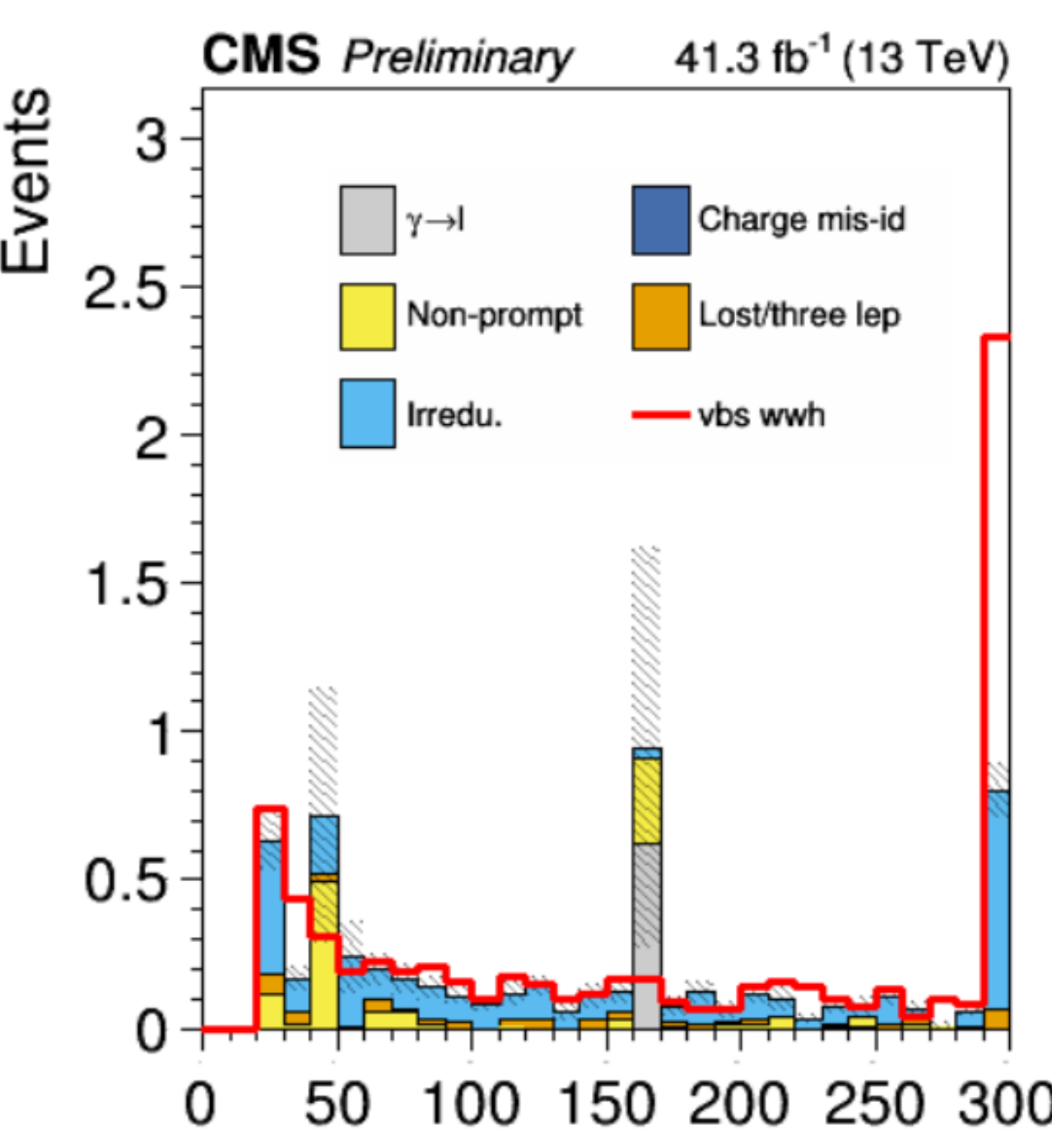

(B) bjet_pt2

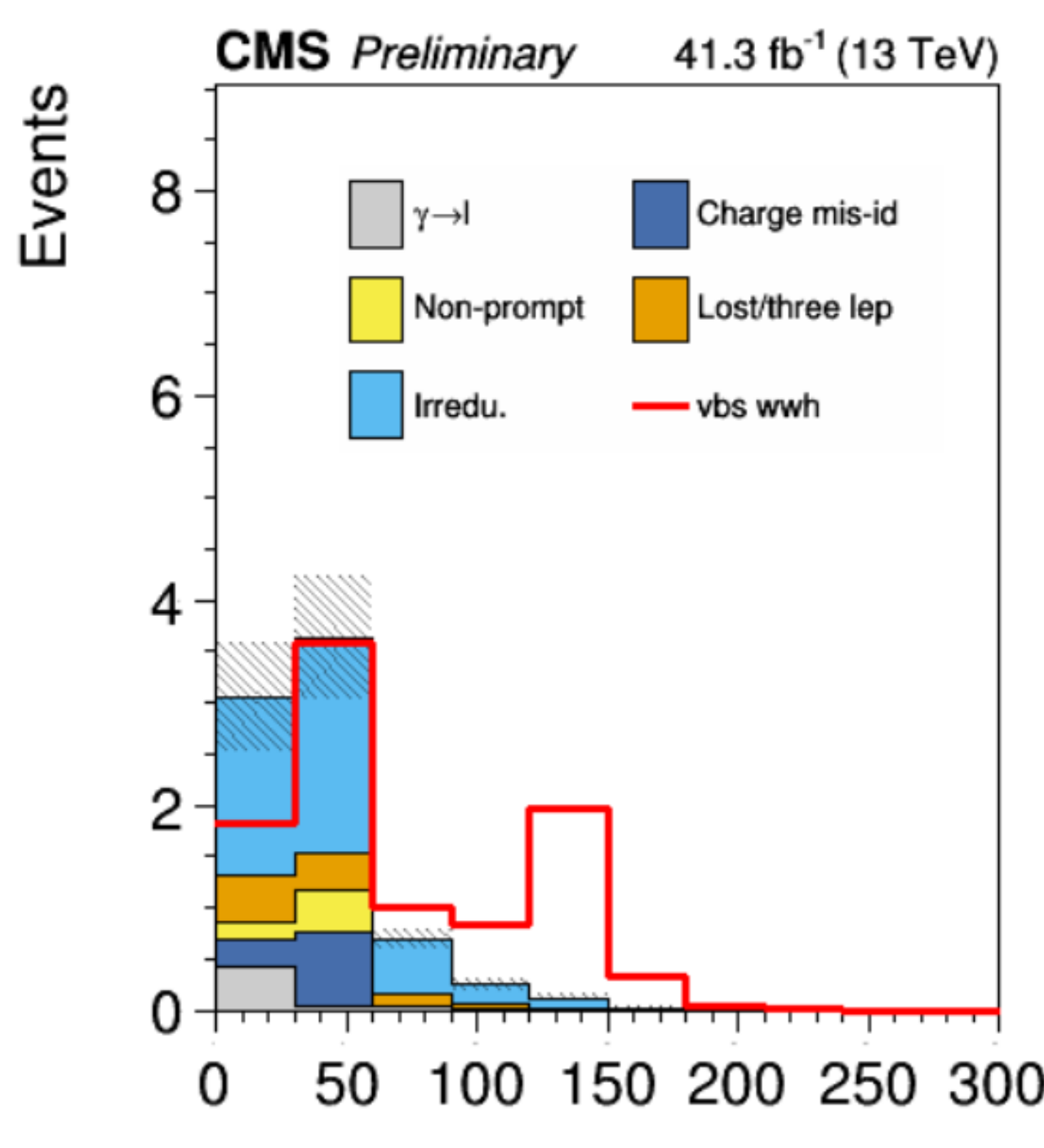

(C) $M b b$

Figure 2: LEFT: Leading b jet transverse momentum. CENTER: sub-leading b jet transverse momentum. RIGHT: combined mass of b jets (shows Higgs mass).

\section{Results}

Figure 2 depicts some of the preliminary distributions from the newly generated sample for the hhh tri-linear $\mathrm{HC}$ process as shown in Figure 1. sample was created on July $30^{\text {th }}$.

- There is a spike at a value of 125 for the combined mass of $b \bar{b}$. This is as expected, since that is the mass of the Higgs boson. This indicates that the sample was generated correctly.

- Due to the same-sign lepton selection, most of the backgrounds are suppressed.

- Expected number of events for this process is extremely low, 50 for 3000 $f b^{-1}$ so signal over background optimization is crucial. Finding this signal will require HL-LHC dataset.

\section{Further Study}

This study is still in progress, as the CMS signal samples were just created. Next steps include:

- Create and study distributions with varied cross-section values determined by the different values of $\kappa_{3}$ (Refer to the table in Figure 3) to find which values agrees the most with the data.

- Further understanding of the signal samples as well as a HL-LHC dataset to extract meaningful information from them.

- Given the complexity of the final states, advanced machine learning techniques are expected to have a crucial role in improving our analysis. [1]

Figure 3: The Different Predicted Cross-Sections for values of $\kappa_{3}$.

${ }^{\star}$ This is preliminary, and cross-section values are still under investigation. $W^{ \pm} W^{ \pm} j j h, C_{1}=0.020322$.

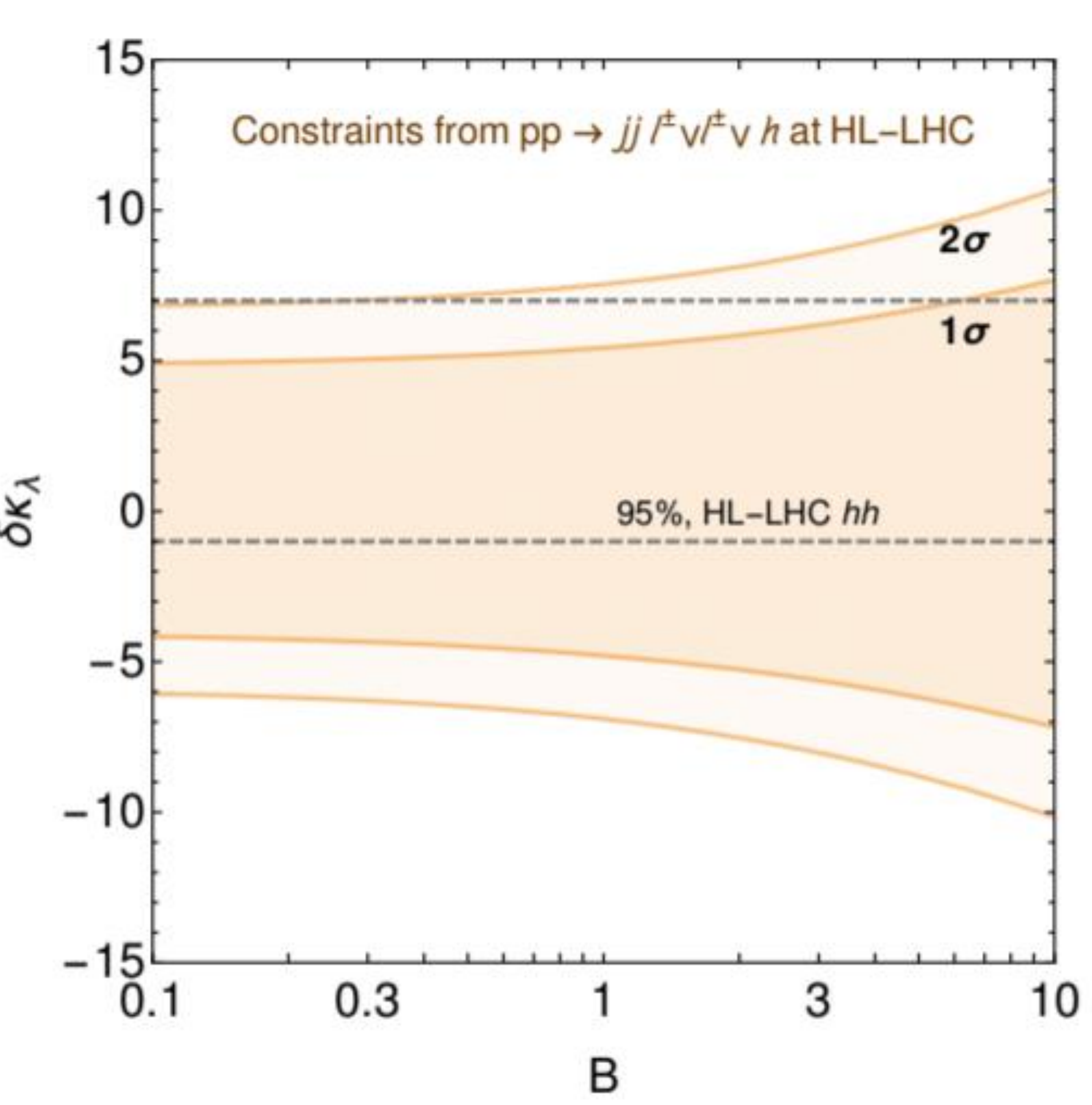

Figure 4: Plot shows sensitivity on modifications to the Higgs Tri-linear (shaded) and from measurements of Higgs Couplings (95\% CL, dashedlines) B controls additional backgrounds. [1]

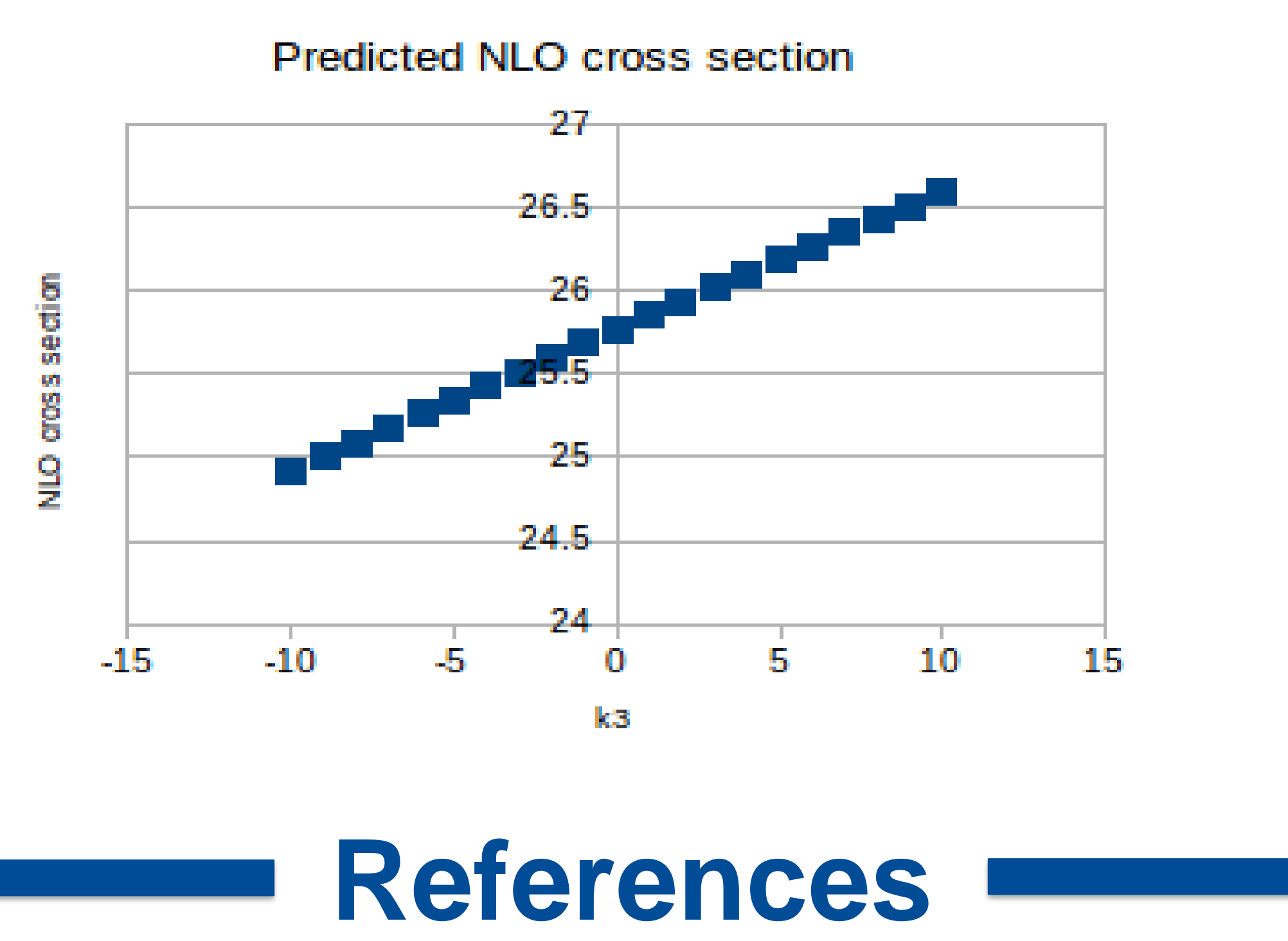

[1] Henning B., \& Lombardo D., \& Riemban M., \& Riva F. (2018), arXiv:1812.09299v1 [hep-ph].

[2] Maltoni, F., \& Pagani D., \& Shivaji A., \& Zhao X. (2018), arXiv:1709.08649v2 [hep-ph]. [3] CERN (2019), The Higgs Boson, Retrieved from https://home.cern/science/phy sics/higgs-boson. 